\section{General Relativity Requires Absolute Space and Time}

\author{
Rainer W. Kühne \\ Lechstr. 63, 38120 Braunschweig, Germany
}

We examine two far-reaching and somewhat heretic consequences of General Relativity. (i) It requires a cosmology which includes a preferred rest frame, absolute space and time. (ii) A rotating universe and time travel are strict solutions of General Relativity.

\section{Space and Time Before General Relativity}

According to Aristotle, the Earth was resting in the centre of the universe. He considered the terrestrial frame as a preferred frame and all motion relative to the Earth as absolute motion. Space and time were absolute [1].

In the days of Galileo the heliocentric model of Copernicus [2] was valid. The Sun was thought to be resting within the centre of the universe and defining a preferred frame. Galileo argued that only relative motion was observed but not absolute motion. However, to fix motion he considered it as necessary to have not only relative motion, but also absolute motion [3].

Newton introduced the mathematical description of Galileo's kinematics. His equations described only relative motion. Absolute motion did not appear in his equations 断.

This inspired Leibniz to suggest that absolute motion is not required by the classical mechanics introduced by Galileo and Newton 5 .

Huyghens introduced the wave theory of light. According to his theory, light waves propagate via oscillations of a new medium which consists of very tiny particles, which he named aether particles. He considered the rest frame of the luminiferous aether as a preferred frame [6].

The aether concept reappeared in Maxwell's theory of classical electrodynamics [7]. Faraday [8] unified Coulomb's theory of electricity [9] with
Ampère's theory of magnetism [10]. Maxwell unified Faraday's theory with Huyghens' wave theory of light, where in Maxwell's theory light is considered as an oscillating electromagnetic wave which propagates through the luminiferous aether of Huyghens.

We all know that the classical kinematics was replaced by Einstein's Special Relativity [11. Less known is that Special Relativity is not able to answer several problems that were explained by classical mechanics.

According to the relativity principle of Special Relativity, all inertial frames are equivalent, there is no preferred frame. Absolute motion is not required, only the relative motion between the inertial frames is needed. The postulated absence of an absolute frame prohibits the existence of an aether 11 .

According to Special Relativity, each inertial frame has its own relative time. One can infer via the Lorentz transformations 12] on the time of the other inertial frames. Absolute space and time do not exist. Furthermore, space is homogeneous and isotropic, there does not exist any rotational axis of the universe.

It is often believed that the Michelson-Morley experiment 13] confirmed the relativity principle and refuted the existence of a preferred frame. This believe is not correct. In fact, the result of the Michelson-Morley experiment disproved the existence of a preferred frame only if Galilei invariance is assumed. The experiment can be completely explained by using Lorentz invariance alone, the relativity principle is not required.

By the way, the relativity principle is not a phenomenon that belongs solely to Special Relativity. According to Leibniz it can be applied also to classical mechanics.

Einstein's theory of Special Relativity has three problems.

(i) The space of Special Relativity is empty. There are no entities apart from the observers and the observed objects in the inertial frames. By contrast, the space of classical mechanics can be filled with, say, radiation or turbulent fluids.

(ii) Without the concept of an aether Special Relativity can only describe but not explain why electric and magnetic fields oscillate in propagating light waves.

(iii) Special Relativity does not satisfy the equiva- 
lence principle 14] of General Relativity, according to which inertial mass and gravitational mass are identical. Special Relativity considers only inertial mass.

Special Relativity is a valid approximation of reality which is appropriate for the description of most of the physical phenomena examined until the beginning of the twenty-first century. However, the macroscopic properties of space and time are better described by General Relativity.

\section{General Relativity: Abso- lute Space and Time}

In 1915 Einstein presented the field equations of General Relativity [15 and in 1916 he presented the first comprehensive article on his theory [16]. In a later work he showed an analogy between Maxwell's theory and General Relativity. The solutions of the free Maxwell equations are electromagnetic waves while the solutions of the free Einstein field equations are gravitational waves which propagate on an oscillating metric [17. As a consequence, Einstein called space the aether of General Relativity [18. However, even within the framework of General Relativity do electromagnetic waves not propagate through a luminiferous aether.

Einstein applied the field equations of General Relativity on the entire universe [19]. He presented a solution of a homogeneous, isotropic, and static universe, where the space has a positive curvature. This model became known as the Einstein universe. However, de Sitter has shown that the Einstein universe is not stable against density fluctuations 20 .

This problem was solved by Friedmann and Lemaitre who suggested a homogeneous and isotropic expanding universe where the space is curved 21].

Robertson and Walker presented a metric for a homogeneous and isotropic universe 22. According to Gödel this metric requires an absolute time [23. In any homogeneous and isotropic cosmology the Hubble constant [24] and its inverse, the Hubble age of the universe, are absolute and not relative quantities. In the Friedmann-Lemaitre universe there exists a relation between the actual age of the universe and the Hubble age.

According to Bondi and Gold, a preferred motion is given at each point of space by cosmological observations, namely the redshift-distance relation generated by the Hubble effect. It appears isotropic only for a unique rest frame [25].

I argued that the Friedmann-Lemaitre universe has a finite age and therefore a finite light cone. The centre-of-mass frame of this Hubble sphere can be regarded as a preferred frame [26].

After the discovery of the cosmic microwave background radiation by Penzias and Wilson [27, it was predicted that it should have a dipole anisotropy generated by the Doppler effect by the Earth's motion. This dipole anisotropy was predicted in accordance with Lorentz invariance [28 and later discovered experimentally [29]. Peebles called these experiments "aether drift experiments" 30.

The preferred frames defined by the RobertsonWalker metric, the Hubble effect, and the cosmic microwave background radiation are probably identical. In this case the absolute motion of the Sun was determined by the dipole anisotropy experiments of the cosmic microwave background radiation to be $(371 \pm 1) \mathrm{km} / \mathrm{s}$.

I suggested that this aether drift can give rise to local physical effects. I introduced the theory of quantum electromagnetodynamics [26]. It is a generalization of quantum electrodynamics 31. which includes Dirac's magnetic monopoles 32 and two kinds of photons, Einstein's electric photon [33] and Salam's magnetic photon [34]. I predicted that every light source which emits electric photons does emit also magnetic photons. The ratio between the interaction cross-sections of the magnetic photon and the electric photon shall depend on the aether drift of the laboratory. The results of recent experiments to test my theory may be interpreted as preliminary evidence for these magnetic photon rays. These experiments were performed in Vienna/Austria by Alipasha Vaziri in February 2002 and in Madison/Wisconsin by Roderic Lakes in March and June 2002.

\section{General Relativity: Ro- tating Universe and Time Travel}

It is well-known that planets, stars, and galaxies rotate. So Lanczos and Gamow speculated that the entire universe may rotate and that the rotating 
universe might have generated the rotation of the galaxies 35].

Gödel was the first to show that a rotating universe is a strict solution of Einstein's field equations for a homogeneous and anisotropic universe. He considered a non-expanding universe and has shown that it allows closed time-like curves, i.e. time-travel. He predicted that the original order of the rotation axes of galaxies was parallel to the universal rotation axis 23 .

Raychaudhuri presented a model for an expanding and rotating universe which is a generalization of both the Friedmann-Lemaitre universe and the Gödel universe. This cosmology, too, includes closed time-like curves [36].

Possibly, the Raychaudhuri universe did not start from a singularity (big bang), but from a closed time-like curve, i.e. from a time-machine.

Gregory, Thompson, and Tifft discovered that the distribution of the rotation axes for both the spiral and ellipsoid galaxies of the filament-like Perseus-Pisces supercluster is bimodal. One of the peaks is roughly aligned with the major axis of the supercluster while the second peak is roughly $90^{\circ}$ from the first [37. This anisotropic distribution cannot be explained by conventional models of galaxy-formation. Therefore I suggested that this might be a remnant of the original aligned distribution of galactic rotation axes generated by a rotating universe [38].

A rotating universe with both vorticity and shear would generate an anisotropy of the cosmic microwave background radiation. Collins and Hawking were able to set tight bounds on this effect 39 . However, Korotky and Obukhov showed that the generation of this anisotropy is an effect of shear and not of vorticity alone. So the observed isotropy of the cosmic microwave background radiation does not contradict the idea of a rotating universe, where the rotation period could be as high as the Hubble age of the universe 40.

There is some discussion whether General Relativity could allow local time-machines. Carter has shown that the Kerr metric 41] of rotating spherical bodies can generate closed time-like curves 42]. This inspired Tipler to investigate a rapidly rotating cylinder with $100 \mathrm{~km}$ length, $15 \mathrm{~km}$ radius, $10^{14} \mathrm{~g} / \mathrm{cm}^{3}$ density, and a rotational speed of $70 \%$ of the speed of light. This object yielded closed timelike curves 433. However, until now it has not been proved that an observer outside the gravitational field would also see time-travel.

To conclude, General Relativity requires a cosmology which includes a preferred frame, absolute space and time and which may include a rotating universe and time-travel. Such a universe may have originated not from a singularity (big bang), but from a closed time-like curve (time-machine).

\section{References}

[1] Aristotle, De caelo (4th century BC).

[2] N. Copernicus, De revolutionibus orbium coelestium (1543).

[3] G. Galilei, Discorsi e dimostrazioni matematiche intorno a due nuove scienze attenenti alla meccanica ed i movimente locali (Leida, Elsevier, 1638).

[4] I. Newton, Philosophiae naturalis principia mathematica (London, 1687).

[5] G. W. Leibniz, Third letter to S. Clarke (1716).

[6] C. Huyghens, Traité de la lumière (1690).

[7] J. C. Maxwell, A Treatise on Electricity and Magnetism (Oxford, Clarendon Press, 1873).

[8] M. Faraday, Experimental Researches in Electricity, Vol. I (London, Taylor and Francis, 1839).

M. Faraday, Experimental Researches in Electricity, Vol. II (London, Richard and John Edward Taylor, 1844).

M. Faraday, Experimental Researches in Electricity, Vol. III (London, Taylor and Francis, 1855).

[9] C. A. Coulomb, Hist. Mém. l'Acad. R. Sci., p. 569 (1785).

C. A. Coulomb, Hist. Mém. l'Acad. R. Sci., p. 578 (1785).

C. A. Coulomb, Hist. Mém. l'Acad. R. Sci., p. $612(1785)$.

C. A. Coulomb, Hist. Mém. l'Acad. R. Sci., p. 67 (1786).

[10] A.-M. Ampère, Ann. Chim. Phys. 15, 59 (1820).

A.-M. Ampère, Ann. Chim. Phys. 15, 170 (1820). 
[11] A. Einstein, Ann. Phys. (Leipzig) 17, 891 (1905).

[12] J. Larmor, Aether and Matter (Cambridge, University Press, 1900).

H. A. Lorentz, Proc. R. Acad. Amsterdam 6, 809 (1904).

[13] A. A. Michelson, Am. J. Sci. 22, 120 (1881). A. A. Michelson and E. W. Morley, Am. J. Sci. 34, 333 (1887).

[14] A. Einstein, Ann. Phys. (Leipzig) 38, 355 (1912).

[15] A. Einstein, S.-B. Preuss. Akad. Wiss., p. 844 (1915).

[16] A. Einstein, Ann. Phys. (Leipzig) 49, 769 (1916).

[17] A. Einstein, S.-B. Preuss. Akad. Wiss., p. 154 (1918).

[18] A. Einstein, Äther und Relativitätstheorie (Berlin, Springer-Verlag, 1920).

[19] A. Einstein, S.-B. Preuss. Akad. Wiss., p. 142 (1917).

[20] W. de Sitter, Konin. Ned. Akad. Wetenschappen 19, 1217 (1917).

[21] A. Friedmann, Z. Phys. 10, 377 (1922).

A. Friedmann, Z. Phys. 21, 326 (1924).

G. Lemaitre, Ann. Soc. Sci. Brux. 47, 49 (1927).

[22] H. P. Robertson, Astrophys. J. 82, 284 (1935). A. G. Walker, Proc. London Math. Soc. 42, 90 (1936).

[23] K. Gödel, Rev. Mod. Phys. 21, 447 (1949).

[24] E. P. Hubble, Proc. Nat. Acad. Sci. 15, 168 (1929).

[25] H. Bondi and T. Gold, Nature 169, 146 (1952).

[26] R. W. Kühne, Mod. Phys. Lett. A 12, 3153 (1997).

[27] A. A. Penzias and R. W. Wilson, Astrophys. J. 142, 419 (1965).
[28] P. J. E. Peebles and D. T. Wilkinson, Phys. Rev. 174, 2168 (1968).

R. N. Bracewell and E. K. Conklin, Nature 219, 1343 (1968).

[29] G. F. Smoot, M. V. Gorenstein, and R. A. Muller, Phys. Rev. Lett. 39, 898 (1977).

[30] P. J. E. Peebles, Physical Cosmology (Princeton, University Press, 1971).

[31] S. Tomonaga, Phys. Rev. 74, 224 (1948).

J. Schwinger, Phys. Rev. 74, 1439 (1948).

J. Schwinger, Phys. Rev. 75, 651 (1949).

J. Schwinger, Phys. Rev. 76, 790 (1949).

R. P. Feynman, Phys. Rev. 76, 749 (1949).

R. P. Feynman, Phys. Rev. 76, 769 (1949).

F. J. Dyson, Phys. Rev. 75, 486 (1949).

F. J. Dyson, Phys. Rev. 75, 1736 (1949).

[32] P. A. M. Dirac, Proc. R. Soc. A 133, 60 (1931).

[33] A. Einstein, Ann. Phys. (Leipzig) 17, 132 (1905).

[34] A. Salam, Phys. Lett. 22, 683 (1966).

[35] C. Lanczos, Z. Phys. 21, 73 (1924).

G. Gamow, Nature 158, 549 (1946).

[36] A. Raychaudhuri, Phys. Rev. 98, 1123 (1955).

[37] S. A. Gregory, L. A. Thompson, and W. G. Tifft, Astrophys. J. 243, 411 (1981).

[38] R. W. Kühne, Mod. Phys. Lett. A 12, 2473 (1997).

[39] S. W. Hawking, Mon. Not. R. Astron. Soc. 142, 129 (1969).

C. B. Collins and S. W. Hawking, Mon. Not. R. Astron. Soc. 162, 307 (1973).

[40] V. A. Korotky and Yu. N. Obukhov, Sov. Phys. JETP 72, 11 (1991).

Yu. N. Obukhov, Gen. Relativ. Grav. 24, 121 (1992).

S. Carneiro and G. A. Mena Marugán, Phys. Rev. D 64, 083502 (2001).

[41] R. P. Kerr, Phys. Rev. Lett. 11, 237 (1963).

[42] B. Carter, Phys. Rev. 174, 1559 (1968).

[43] F. J. Tipler, Phys. Rev. D 9, 2203 (1974). 